levels of natural moisturising factor and cytokines. Questionnaires will be used to investigate the exposure to skin hazards, protective behaviour and knowledge on prevention of OCD. The skin condition of the hands will be assessed by regular clinical examinations and questionnaires. In addition, the same genetic and phenotypic biomarkers will be analysed in a cohort of metal workers affected by OCD. The primary objective is to evaluate if health education is effective in prevention of OCD in metal apprentices. Moreover, the value of different biomarkers to identify individuals at risk for OCD will be assessed.

Results The design of the study and its first results will be presented at the meeting.

Discussion Health education has been shown to be an important key in prevention of OCD. However, intervention studies are necessary to evaluate and improve preventive programmes based on health education. Biomarkers may help to identify individuals at risk and to develop targeted strategies to reduce the burden of OCD.

\section{5d CORRELATING BIOLOGICAL MONITORING FOR PLATINUM WITH DERMAL AND RESPIRATORY EXPOSURE}

Johan du Plessis. Occupational Hygiene and Health Research Initiative, North-West University, Potchefstroom, South Africa

\subsection{6/oemed-2018-ICOHabstracts.254}

Introduction Occupational respiratory exposure to platinum is well established in precious metals refineries (PMR). Soluble platinum causes respiratory sensitisation leading to amongst others occupational asthma and rhinitis. However, several skin symptoms have been reported and the relationship between skin exposure and uptake determined in urine has not been investigated.

Objectives To evaluate the dermal and respiratory exposure of PMR workers to soluble platinum, and to quantify the absorbed platinum concentration excreted via the urine.

Methods Dermal exposure samples were collected on the dominant palm, wrist, neck and forehead using Ghostwipes. Respiratory samples were collected using an Institute of Occupational Medicine inhalable aerosol sampler. Wipe and respiratory samples were analysed according to the MDHS 46/ 2 using inductively coupled plasma-mass spectrometry. The dermal and respiratory exposures of workers from different production areas in two PMRs were measured simultaneously on two consecutive days. Urine samples were collected on the morning of the first day and on the two following mornings. Results The degree of dermal and respiratory exposure varied considerably between workers working in different areas of the refineries. Most workers experienced dermal and respiratory exposure to soluble platinum above the detection limit $(0.005 \mu \mathrm{g})$ with $25 \%$ of the respiratory exposures exceeding the national 8 hour occupational exposure limit of $2 \mu \mathrm{g} / \mathrm{m} 3$. Dermal exposure (average of anatomical positions) were $\leq 6.79 \mu \mathrm{g} / \mathrm{cm} 2$. Urine platinum concentrations ranged between $<0.1$ and $3 \mu \mathrm{g} / \mathrm{g}$ creatinine. Statistically significant positive correlations were established for: (i) average dermal exposure and average respiratory exposure, (ii) average dermal exposure and average platinum urinary concentration, and (iii) average respiratory exposure and average platinum urinary concentration.
Discussion The concentration platinum in the urine of workers is determined by both the dermal and respiratory exposure routes. The skin is as a route of exposure to soluble platinum should be considered.

\section{5e HEALTHY HANDS: HOW TECHNOLOGY CAN SUPPORT A CULTURE OF BEST PRACTICE FOR HAND CARE AMONG NURSES WITH HIGH RISK FOR HAND DERMATITIS}

Maryam Soltanipoor. Coronel Institute of Occupational Health, Academic Medical Centre, Amsterdam, Netherlands

\subsection{6/oemed-2018-ICOHabstracts.255}

Introduction Occupational hand dermatitis (OHD) is a major risk for health care workers (HCW) due to frequent exposure to 'wet work'. With an estimated point prevalence of OHD ranging between $12 \%-30 \%$, nurses are at highest risk. There is a big burden of disease due to chronicity, absenteeism, risk of unemployment and impaired quality of life. Despite evidence and guidelines on the importance of skin care in the prevention of OHD, use of hand creams during work is reported to be very low. New preventive strategies are obviously needed.

Methods A cluster randomised controlled trial has been initiated in nurses performing wet work, with a follow-up of 18 months. Twenty wards have been recruited to include 504 participating nurses in the study at baseline. Nurses in the control and intervention wards received health education about optimal hand care every three months from baseline. The intervention wards were additionally provided with hand creams in dispensers equipped with the electronic system for the continuous registration of cream consumption. Regular feedback on skin care performance at ward level was provided by using posters. At baseline and 12 months clinical examination of the skin condition was done using the Hand Eczema Severity Index (HECSI score). In addition, stratum corneum samples were collected for analysis of epidermal levels of Natural Moisturising Factor (NMF), as an early biomarker of skin barrier damage. All participants completed questionnaires regarding exposure to wet work and skin protective behaviour during the study period.

Results The design of the study and a summary of the main results will be presented at the session

Discussion This trial will assess whether provision of hand creams coupled with continuous monitoring and regular feedback on its consumption improves skin condition in healthcare workers

\section{TAKING STOCK OF NEW DEVELOPMENTS IN THE PREVENTION OF OCCUPATIONAL AND ENVIRONMENTAL DERMATOSES}

Swen Malte John. Dept. Dermatology, Environmental Medicine, Health Theory, University of Osnabrueck, Germany

\subsection{6/oemed-2018-ICOHabstracts.256}

Aim of special session Showcase that with innovative approaches sustainable prevention can be achieved to decrease cases of occupational skin diseases

OSD represent up to $35 \%$ of notified occupational illnesses. Prolonged absence from work due to OSD jeopardizes 
competitiveness especially of small and medium sized enterprises, where OSD-incidence peaks. For affected individuals, the chronic course of OD may result in job loss, precarious or unemployment. A joint coordinated approach to patient management as well as standardization of diagnostics, therapy, protective equipment as well as workers' education is lacking in Europe and other parts of the World. Important sustainable prevention strategies applied in various countries will be presented during this session.

\section{8 a OSD PREVENTION: WHY? A EUROPEAN AND GLOBAL PERSPECTIVE}

Swen Malte John. Dept. Dermatology, Environmental Medicine, Health Theory, University of Osnabrueck, Germany

\subsection{6/oemed-2018-ICOHabstracts.257}

Introduction In Europe, occupational skin diseases (OSD) mainly irritant and allergic contact dermatitis of the hands constitute currently up to $40 \%$ of all work-related illnesses causing extensive suffering for affected workers. Due to emerging new workplace hazards and demographic change OSD are likely to increase over the next years. The annual costs incurred by OSD are estimated to exceed by far 5 billion $€$ in the EU due to medical treatment, sick leave and loss of productivity.

Results Recent studies have demonstrated that dermatological intervention can save OSD patients' health and jobs, and avoid individual suffering as well as reduce costs for society. A longitudinal study carried out in Germany with severely OSD affected workers showed that due to an interdisciplinary in-patient prevention scheme $80 \%$ of patients were still working 3 years after the measure and sick leave was sustainably reduced (total cohort: $N=1,409$ ). Similar results were obtained from a randomized nationwide follow up study of initial cases of contact dermatitis receiving outpatient skin protection seminars and regular outpatient treatment by the local dermatologists $(\mathrm{N}=1,600)$. However, as yet, insurance systems in many countries do neither enable specific dermatological intervention nor specific preventive measures. Since 2010 the EADV 'healthy skin @work' campaign aims at raising awareness at the primary prevention level, and also to improve options for medical care for affected individuals, and coordinate scientific efforts. This dermatological initiative is also an official partner of the 'Healthy workplaces campaign' by the European Agency for Safety and Health at Work (EU OSHA).

Discussion In various European countries OSD specific centers have been or are being implemented (e.g. Austria and Denmark) geared at applying specifically tailored measures for OSD patients. In Germany on the other hand, where since 2009 the 'Week of Occupational skin Diseases (WOOD)' has been carried out, a 30\% increase of OSD notifications could be observed, which will help tackle the under-reporting. At the same time, costs for job-retraining have substantially decreased due to earlier and more effective preventive intervention. 1618b THE OCCUPATIONAL DISEASE ACTION PLAN: ONTARIO, CANADA TAKES A STEP FORWARD

Linn Holness. Centre for Research Expertise in Occupational Disease, University of Toronto and St Michael's Hospital, Ontario, Canada

\subsection{6/oemed-2018-ICOHabstracts.258}

Introduction Government legislation, regulation and policy are important components of workplace prevention. While many jurisdictions have legislation and regulations that provide direction for workplace prevention, it is also important the government promotes workplace prevention in priority areas. In Ontario the focus has traditionally been on accidents and ergonomic issues. In 2016 the Province of Ontario, Canada initiated an Occupational Disease Action Plan (ODAP) to move the prevention of occupational disease into priority focus. The objective of this session is to describe the process leading to the development and implementation of the ODAP with respect to skin allergens and irritants.

Methods The Prevention Office of the Ontario Ministry of Labour held a meeting bringing together of occupational disease research and data experts and occupational health and safety (OHS) system partners to review and recommend where prevention efforts should be focused. Two lists of potential exposure and disease priorities were developed. A Reference Group was formed of OHS system partners that created a propriety ranking of areas for focus. An Implementation Team and Working Groups were formed to carry each priority area forward.

Results The five priority areas identified included general occupational disease awareness and skin and lung allergens and irritants. A working group for Allergens and Irritants is initially identifying priority allergens and irritants and will then move forward with awareness, resource and training development.

Discussion The development and implementation of the ODAP will see increased government and OHS system partner focus on occupational disease including skin allergens and irritants. Following prioritization of key skin allergens and irritants, resources and training opportunities will be reviewed and gaps filled. In addition, Ministry of Labour operations staff will ensure that the ODAP priorities are included in inspections and other activities.

\section{$1618 \mathrm{C}$ EFFECTIVENESS OF SECONDARY PREVENTION FOR OCCUPATIONAL DERMATITIS: THE EXPERIENCE IN TRIESTE HOSPITALS}

Francesca Larese Filon. Unit of Occupational Medicine, Dept. of Medical Sciences, University of Trieste, Italy

\subsection{6/oemed-2018-ICOHabstracts.259}

Introduction Primary and secondary prevention for occupational contact dermatitis is a challenge for the occupational physician: educational training and the avoidance of contact with irritant and allergic substances permits a reduction of incidence cases and days of work lost for a disease that is the third in frequency among workers in industrialized countries. Methods Workers with a diagnosis of occupational hand dermatitis were invited to a training course on prevention of skin 